\title{
Posterior versus Anterior Approach Surgery for Thoracolumbar Spinal Tuberculosis
}

\author{
Zhigang Huang, Jing Liu and Ke Ma
}

\begin{abstract}
The aim of this study was to compare clinical effect of posterior approach and anterior approach surgery for patients with thoracolumbar spinal tuberculosis. It was a descriptive, analytical study carried out at the Third People's Hospital of Shenzhen City, China, from March 2012 to March 2015. A total of 84 patients with thoracolumbar spinal tuberculosis were randomly divided into group A and group B, each group having 42 patients. Group A was treated with posterior approach surgery, while group B was treated with anterior approach surgery. The results showed that operation time, bleeding condition, length of stay of group A were lower than those of group $B(p<0.001, p<0.001$ and $p=0.013$, respectively). Compared with group $B$, the Cobb angle of group A after surgery was lower $(p<0.001)$. At the 28th day after treatment, the serum bone alkaline phosphatase (BAP), bone gla protein (BGP), procollagen type I carboxy terminal peptide (CTX) levels of group A were lower than those of group $B(p<0.001, p<0.001$ and $p=0.002$, respectively). As compared to anterior approach surgery, posterior approach surgery was an effective method to treat thoracolumbar spinal tuberculosis and reduce serum levels of BAP, BGP and CTX.
\end{abstract}

Key Words: Posterior approach, Anterior approach, Thoracolumbar spinal tuberculosis.

Spinal tuberculosis is the most common osteoarticular tuberculosis caused by tuberculous mycobacteria. ${ }^{1}$ At present, surgery is the optimal treatment method for such disease, with purpose of removing tuberculosis focus, correcting kyphosis, and restoring the stability of spine. There are still some disputes on the choice of surgical method for thoracolumbar spinal tuberculosis. Some scholars advocate one-stage posterior focus clearance with internal fixation, and argue that the treatment of lumbar vertebral tuberculosis with single posterior focus clearance and lumbar interbody fusion has the advantages of safety and high efficiency. ${ }^{2}$ However, some scholars advocate lumbar anterior focus clearance and fusion, and claim that it can not only make full and direct decompression, but also achieve the goal of fixation, fusion and maintenance of spinal stability. ${ }^{3}$ Bone alkaline phosphatase (BAP), bone gla protein (BGP), and procollagen type I collagen carboxy terminal peptide (CTX) are important serum bone markers. They can be used to evaluate clinical therapeutic effect of spinal tuberculosis.

The aim of this study was to compare clinical outcomes of surgical management by posterior approach and anterior approach for thoracolumbar spinal tuberculosis.

This descriptive, analytical study was carried out at the Third People's Hospital of Shenzhen City, China, from

Department of Orthopedics, The Third People's Hospital of Shenzhen City, 518112, China

Correspondence: Ke Ma, Department of Orthopedics,

The Third People's Hospital of Shenzhen City, 518112 China

E-mail:sun2012mo@163.com

Received: April 15, 2017; Accepted: September 20, 2018.
March 2012 to March 2015. A total of 84 patients with thoracolumbar spinal tuberculosis, confirmed by clinical manifestations, clinical signs, imaging characteristics, laboratory examinations and histopathological manifestations, were included. Patients with the medical history of thoracolumbar operation, congenital vertebral anomalies etc., that might interfere with postoperative assessment, were excluded.

All patients were randomly divide into group $A$ and group B, each group having 42 patients. Group A was applied with posterior approach surgery; namely, combination therapy of posterior approach, auto-bone graft fusion, internal fixation. Group B was treated with anterior approach surgery; namely, combination therapy of anterior approach, auto-bone graft fusion, posterior internal fixation.

Operation time, bleeding condition, length of stay, and Cobb angle in two groups were compared. At the $28^{\text {th }}$ day after surgery, BAP, BGP and CTX in serum were compared. Collected data were analysed using independent t-test by SPSS 20. Statistical significance was attributed to $p<0.05$.

Eighty-four patients, including $44(52.38 \%)$ males and $40(47.62 \%)$ females, aged within 19-73 years, with average age of $56.25 \pm 3.57$ years. Of them, there were $50(59.52 \%)$ patients with pure thoracic tuberculosis, 20 (23.81\%) patients with pure spinal tuberculosis, and 14 $(16.67 \%)$ patients with both thoracic and spinal tuberculosis.

Operation time, bleeding condition, length of stay of group $A$, were lower than those of group $B(p<0.001$, $p<0.001$ and $p=0.013$, respectively). After surgery, Cobb angles of group $A$ was lower than that of group $B$ 
Table I: Comparison of clinical outcomes between two groups.

\begin{tabular}{l|c|c|c|c|c|c|c|c}
\hline Groups & $\mathrm{n}$ & Operation time $(\mathrm{min})$ & Bleeding condition $(\mathrm{mL})$ & Length of stay $(\mathrm{d})$ & Cobb angles $\left(^{\circ}\right)$ & $\mathrm{BAP}(\mathrm{ng} / \mathrm{mL})$ & $\mathrm{BGP}(\mathrm{ng} / \mathrm{mL})$ & $\mathrm{CTX}(\mathrm{ng} / \mathrm{mL})$ \\
\hline Group A & 42 & $138.64 \pm 5.93$ & $642.37 \pm 31.19$ & $22.73 \pm 4.67$ & $14.66 \pm 4.89$ & $25.44 \pm 0.99$ & $28.27 \pm 0.80$ & $0.29 \pm 0.07$ \\
Group B & 42 & $175.23 \pm 8.77$ & $961.25 \pm 42.07$ & $25.48 \pm 5.26$ & $22.48 \pm 4.56$ & $28.95 \pm 0.76$ & $32.83 \pm 1.02$ & $0.35 \pm 0.10$ \\
\hline $\begin{array}{l}\text { Group A vs. Group B } \\
\text { p-value }\end{array}$ & & $<0.001$ & $<0.001$ & 0.013 & $<0.001$ & $<0.001$ & $<0.001$ & 0.002 \\
\hline
\end{tabular}

$(p<0.001)$. At the 28th day after treatment, BAP, BGP, CTX levels of group $A$ were lower than those of group $B$ $(p<0.001, p<0.001$ and $p=0.002$, respectively, Table I).

Posterior approach surgery can not only effectively reduce bleeding condition, but also effectively shorten operation time and length of stay as compared with anterior approach surgery.

This research showed that appropriate internal fixation could correct kyphosis, reconstruct spine stability, and promote bone graft fusion. The authors found that the curative effect of posterior approach surgery was better than that of anterior approach surgery. The reasons are as follows: First, the posterior approach can directly relieve spinal stenosis, and fully ease intraspinal dura mater and nerve root compression. ${ }^{4}$ Secondly, posterior approach can complete focus clearance, spinal decompression and internal fixation at a time, so patients have good compliance and little mental stress, which has a positive impact on recovery. ${ }^{5}$

The authors' previous study found that BAP and BGP were involved in the occurrence and development of spinal tuberculosis. Studies found that level of serum CTX in patients with spinal tuberculosis was higher than normal healthy population. 6

This research results show that posterior approach surgery can reduce BAP, BGP, CTX levels, improve abnormal bone metabolism, restrain osteoclastic activity, and enhance bone mineralisation.

\section{REFERENCES}

1. Marais S, Roos I, Mitha A, Mabusha SJ, Patel V, Bhigjee AI. Spinal tuberculosis: Clinicoradiological findings in 274 patients. Clin Infect Dis 2018; 67:89-98.

2. Zhang $H$, Sheng B, Tang M, Guo C, Liu S, Huang S, et al. Onestage surgical treatment for upper thoracic spinal tuberculosis by internal fixation, debridement, and combined interbody and posterior fusion via posterior-only approach. Eur Spine J 2013; 22:616-23.

3. Wang Z, Wu Q, Geng G. Anterior debridement and bone grafting with posterior single-segment internal fixation for the treatment of mono-segmental spinal tuberculosis. Injury 2013; 44:253-7.

4. D'souza AR, Mohapatra B, Bansal ML, Das K. Role of posterior stabilization and transpedicular decompression in the treatment of thoracic and thoracolumbar TB: A retrospective evaluation. Clin Spine Surg 2017; 30:E1426-33.

5. Jain A, Jain RK, Kiyawat V. Evaluation of outcome of transpedicular decompression and instrumented fusion in thoracic and thoracolumbar tuberculosis. Asian Spine J 2017; 11:31-6.

6. An JY, Li DW, Cui X, Ma YZ. Correlation between spine tuberculosis and bone mass. Chin J Bone Joint Surg 2013; 6:145-7. 